\title{
Biomechanical Effects of Implant Materials on Posterior Lumbar Interbody Fusion: Comparison of Polyetheretherketone and Titanium Spacers Using Finite Element Analysis and Considering Bone Density
}

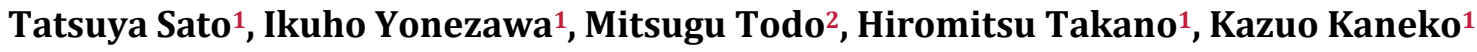 \\ ${ }^{1}$ Department of Orthopaedic Surgery, Juntendo University, Tokyo, Japan; ${ }^{2}$ Renewable Energy Center, Research \\ Institute for Applied Mechanics, Kyushu University, Kasuga, Japan
}

Correspondence to: Ikuho Yonezawa, yoza@juntendo.ac.jp

Keywords: Posterior Lumbar Interbody Fusion, Biomechanics, Finite Element Analysis, Cage, Polyetheretherketone, Titanium, Osteoporosis

Received: March 12, $2018 \quad$ Accepted: April 22, $2018 \quad$ Published: April 25, 2018

Copyright ( $) 2018$ by authors and Scientific Research Publishing Inc.

This work is licensed under the Creative Commons Attribution International License (CC BY 4.0).

http://creativecommons.org/licenses/by/4.0/

\section{(c) (1) Open Access}

\section{ABSTRACT}

Few biomechanical data exist regarding whether the polyetheretherketone (PEEK) spacer or titanium spacer is better for posterior lumbar interbody fusion (PLIF). This study evaluated the biomechanical influence that these types of spacers with different levels of hardness exert on the vertebra by using finite element analysis including bone strength distribution. To evaluate the risk of spacer subsidence for PLIF, we built a finite element model of the lumbar spine using computed tomography data of osteoporosis patients. Then, we simulated PLIF in L3/4 and built models with the hardness of the interbody spacer set as PEEK and titanium. Bones around the spacer were subjected to different load conditions. Then, fracture elements and some stress states of the two modalities were compared. In both models of PLIF simulation, fracture elements and stress were concentrated in the bones around the spacer. Fracture elements and stress values of the model simulating the PEEK spacer were significantly smaller compared to those of the titanium simulation model. For PLIF of osteoporotic vertebrae, this suggested that the PEEK spacer is in a mechanical environment less susceptible to subsidence caused by microfractures of bone tissue and bone remodeling-related fusion aspects. Therefore, PEEK spacers are biomechanically more useful.

\section{INTRODUCTION}

Posterior lumbar interbody fusion (PLIF) is a technique that uses a bone graft for stabilization in the 
lumbar vertebrae, thereby providing bone fusion [1]. In recent years, PLIF, which uses posterior fixation with an interbody spacer filled with a bone graft and pedicle screw (PS), has been widely adopted as a treatment option that provides stability until spinal fusion occurs [2-5]. This contributed to a higher fusion rate. However, in the vertebrae of the elderly, for whom PLIF is usually applied, complications resulting from instrumentation, such as spacer subsidence, loosening screws, and degeneration of the adjacent intervertebral disk, have become prevalent [6-8]. Currently, spacers with many different designs intended to provide early-stage stability necessary for interbody fusion have been commercialized. Those spacers are mainly made of polyetheretherketone (PEEK), titanium alloy, or pure titanium (Ti). However, clinical evidence regarding whether the PEEK or Ti spacer is more suitable for PLIF is limited [9]. Therefore, surgeons choose the type of spacer based on their own experience. Furthermore, biomechanical factors are also important when comparisons between those spacers are performed to determine the significance associated with durability, stress shielding, microdamage, and bone remodeling. Although this is a highly important subject, there is little biomechanical evidence regarding such biomechanical factors.

One of the established tools used to evaluate the biomechanical parameters of vertebral columns is finite element (FE) analysis [10-14]. Previous authors have compared the mechanical behavior of PEEK and Ti spacers for PLIF using FE models $[13,14]$ created using the theoretical values of morphological models and mechanical properties. However, comparative research involving an aged lumbar spinal specimen and FE models indicated that FE models with material properties created from theoretical values are insufficient and that inputting morphological and structural changes of the vertebrae caused by aging into the FE models is important [15]. Therefore, it is questionable whether the results will be the same as those of past FE analysis reports using theoretical morphological models and mechanical properties when the influence of aging is considered. Nevertheless, few FE analyses performed using clinical data of morphological models and mechanical properties and considering the influence of osteoporosis among the elderly have been performed.

The objective of this research was to perform a comparative study involving patient-specific FE analysis of the mechanical behavior of the vertebrae during PLIF with PEEK and titanium spacers by using the FE model created from computed tomography (CT) images of patients.

\section{MATERIALS AND METHODS}

\subsection{Development of the Intact Model}

To create a patient-specific FE model, we inputted the digital imaging and communications in medicine (DICOM) data of the lumbar spine CT images of a 72-year-old woman diagnosed with osteoporosis into a three-dimensional FE analysis program called Mechanical Finder (MF) (version 7.0, Extended Edition; RCCM Co. Ltd., Tokyo, Japan), which has been the most widely used program [16]. We also set the range of interest (ROI) as the second to fifth vertebrae and created a numerical model of the anatomical structure of the vertebral column. According to Keyak et al. [17], MF is capable of not only defining a specific Young's modulus for each of the finite elements from the corresponding bone densities but also performing failure analyses.

The three-dimensional model was divided into tetrahedral solid elements with variable sizes of 0.75 to $10 \mathrm{~mm}$, and $0.2-\mathrm{mm}$ shell elements were also attached to the bone surface to provide structural stability [18]. The numbers of nodes, solid elements, and shell elements were 35,373, 171,538, and 22,724, respectively.

\subsection{Development of the PLIF Models}

PLIF models with posterior fixation were created for the L3 and L4 levels of the intact FE model using the stereolithographic (STL) data of the implants. The implants used were interbody spacers $(8 \mathrm{~mm} \times 22$ $\mathrm{mm}$ : TELAMON-P Medtronic, Memphis, TN, USA), titanium pedicle screws $(6.5 \mathrm{~mm} \times 45 \mathrm{~mm})$, and titanium rods $(5.5 \mathrm{~mm} \times 40 \mathrm{~mm})$. The spacer material was assumed to be PEEK or titanium. The interac- 
tions between implants and the spinal components were as follows: the cage and endplate were in contact with each other, the PS and vertebral body were in contact with each other, and the PS and rod were fixed. The numbers of nodes, solid elements, and shell elements were 78,112, 358,669, and 41,090, respectively. The intact FE model without implants was called LS-INT. The PLIF model with PEEK spacers and the posterior fixation setting was called LS-PEEK. The PLIF model with the titanium spacer setting was called LS-Titanium (Figure 1).

\subsection{Development of the PLIF Models}

MF is a program that is capable of determining the element-specific Young's modulus value from the mean bone density of each finite element using the method of Keyak et al. [17]. Bone density $\rho$ [g/ $\left./ \mathrm{cm}^{3}\right]$ was calculated on the assumption of a linear relationship between CT value [Hounsfield unit (HU)] and $\rho$. Keyak's conversion formula was used to calculate the Young's $(E)[\mathrm{MPa}]$ and yield stress $\left(\sigma_{r}\right)[\mathrm{MPa}]$ of bone from $\rho$, as shown in the following equations [17].

$$
\begin{gathered}
\rho= \begin{cases}0.0(H U \leq-1) & \\
(H U+1.4246) \times \frac{0.001}{1.0580}(-1 \leq H U)\end{cases} \\
E= \begin{cases}0.001 & (\rho=0) \\
33900 \rho^{2.20} & (0<\rho \leq 0.27) \\
5370+469 & (0.27<\rho<0.6) \\
10200 \rho^{2.01} & (0.6 \leq \rho)\end{cases}
\end{gathered}
$$

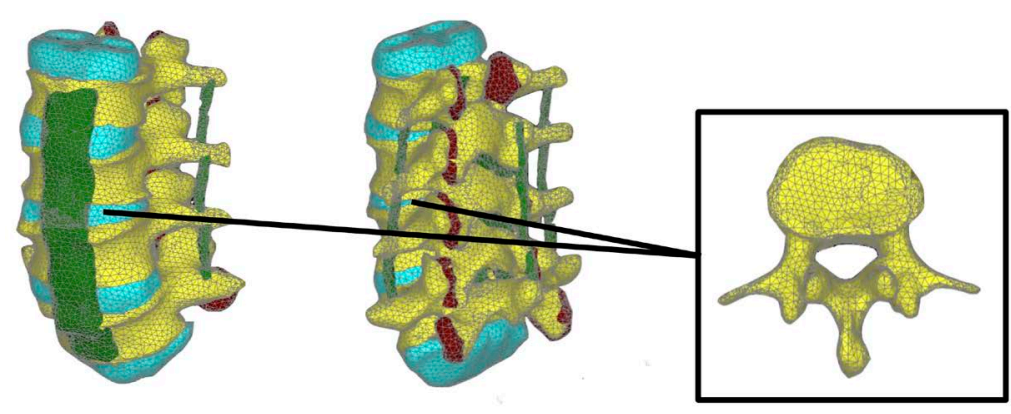

(A)

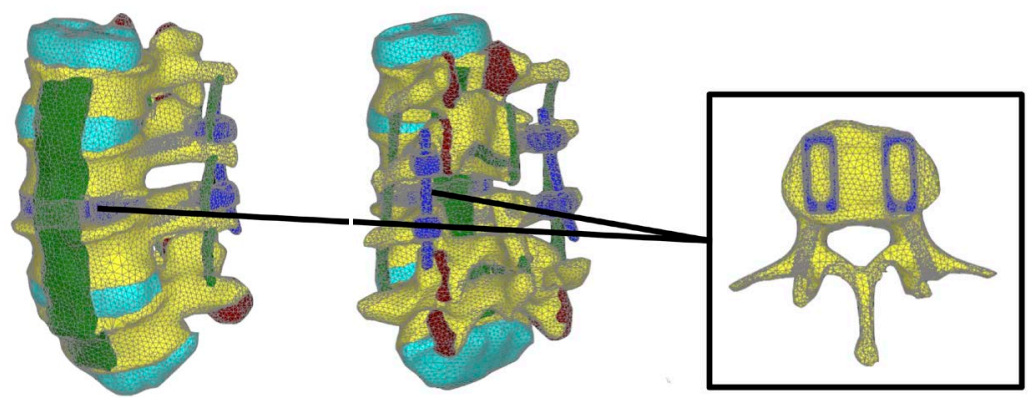

(B)

(C)

Figure 1. Finite element (FE) models. (A) LS-INT: intact FE model; (B) LS-PEEK: FE model of the spine with a polyetheretherketone (PEEK) spacer placed at the L3-4 disc using the posterior approach and posterior instrumentation (PI); (C) LS-Titanium: FE model of the spine with titanium spacer placed at the L3-4 disc using the posterior approach and PI. 


$$
\sigma_{r}=\left\{\begin{array}{l}
1.0 \times 10^{20}(\rho \leq 0.2) \\
137 \rho^{1.88}(0.2<\rho<0.317) \\
114 \rho^{1.72}(0.317 \leq \rho)
\end{array}\right.
$$

Poisson's ratio was fixed at 0.4 [19], and the mechanical properties of each material were obtained from a previous report [20] (Table 1).

To simulate physiological movements of the spine, as following loading conditions of loading were used in the FE models: compression of $400 \mathrm{~N}$, assuming a standing rest position with a vertical load of 400 $\mathrm{N}$; flex-ion and extension, assuming rotational loads of bending forward and backward; and compression of 0 to $1500 \mathrm{~N}$, assuming a posture during which an axial load with increments from 0 to $1500 \mathrm{~N}$ was applied to the lumbar spine. Loads were applied to the superior surface and superior articular facet of L2 vertebra. The in-ferior surface and inferior articular facet of L5 vertebra were fixed in all directions (Figure 2) [20].

\subsection{Static Elastic Analysis during Flexion and Extension}

Static elastic analysis without any damage formation was also performed to evaluate the mechanical behavior of the vertebrae under a relatively low level of loading. The static elastic analysis compared the Drucker-Prager stress distribution of the inferior surface of L3 of each model during flexion and extension.

Table 1. Material properties of the components used in this study. Abbreviations: ALL, anterior longitudinal ligament; PLL, posterior longitudinal ligament; TL, intertransverse ligament; CL, capsular ligaments of facet joints; LF, ligamentumflavum; ISL, interspinous ligament; SSL, supraspinous ligament; PEEK, polyetheretherketone.

\begin{tabular}{ccc}
\hline Component & Young's modulus $(\mathrm{MPa})$ & Poisson ratio \\
Bone & $0.001(\rho=0)$ & \\
& $33,900 \rho^{2.20}(0<\rho \leq 0.27)$ & \\
Ligaments & $5370+469(0.27<\rho<0.6)$ & \\
ALL & $10,200 \rho^{2.01}(0.6 \leq \rho)$ & 0.3 \\
PLL & & 0.3 \\
TL & 20 & 0.3 \\
CL & 20 & 0.3 \\
LF & 59 & 0.3 \\
ISL & 33 & 0.3 \\
SSL & 20 & 0.3 \\
Screw (titanium) & 12 & 0.28 \\
Rod (titanium) & 15 & 0.28 \\
Cage (PEEK) & 110,000 & 0.25 \\
Cage (titanium) & 110,000 & 0.28 \\
Annulus fibrosus & 3600 & 0.45 \\
\hline
\end{tabular}

$\rho\left(\mathrm{g} / \mathrm{cm}^{3}\right)=(\mathrm{HU}+1.4246) \times 0.001 / 1.058(\mathrm{HU}>-1)=0.0(\mathrm{HU} \leq-1)$, where HU represents CT density values in Hounsfield Units. 


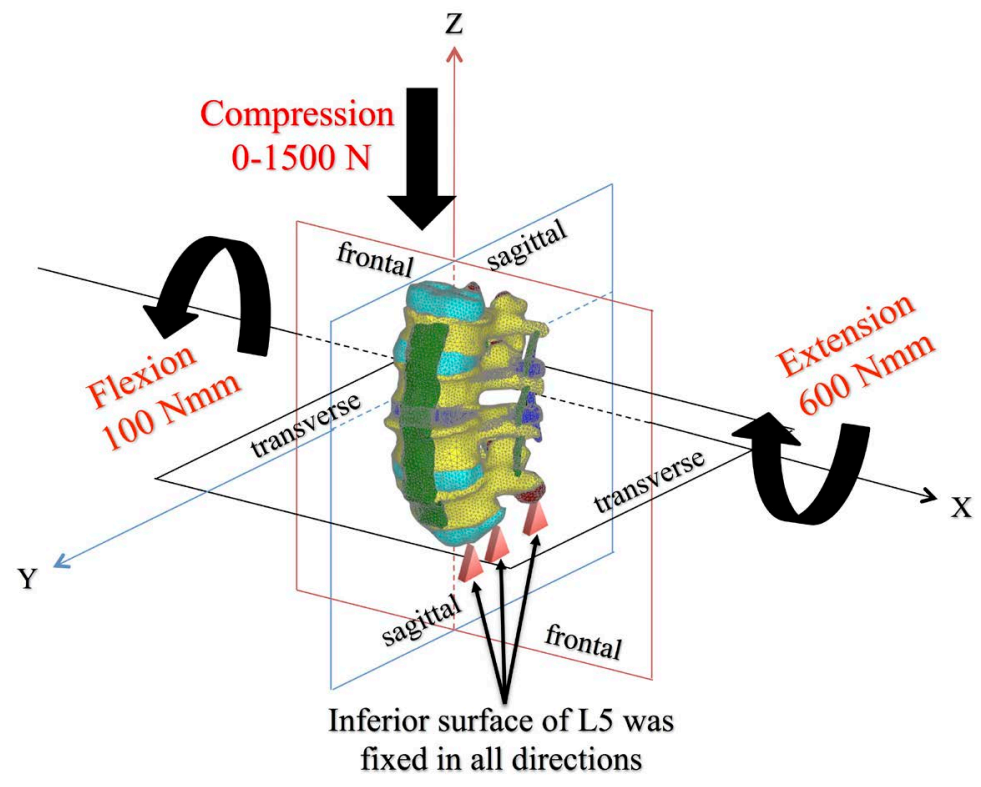

Figure 2. Loads and boundary conditions. Loads were applied to the superior surface and superior articular facet of L2 vertebra. The inferior surface and inferior articular facet of L5 vertebra were fixed in all directions.

To evaluate the mechanical behavior around the implant under each load condition (flexion and extension), the bones around the left spacer in L3 were divided into 20 equal areas (areas 1 to 20, each with thickness, width, and depth of $1.5 \mathrm{~mm}, 15 \mathrm{~mm}$, and $5 \mathrm{~mm}$, respectively), and the mean values of Drucker-Prager stress, strain energy density (SED), and minimum principal strain of each area were compared between the two models (Figure 3).

Statistical software (IBM SPSS Statistics 21; IBM Japan, Tokyo, Japan) was used for the analyses. A t-test was used for normally distributed continuous variables. A $p$-value $<0.05$ was considered statistically significant.

\subsection{Nonlinear Fracture Analysis}

When external forces in a vertical direction, such as during a fall, act on the human body, the high axial load is expected to be exerted on the spine. Furthermore, micro-damage formation is sometimes expected in the spine because of such high external loading, especially for older patients with osteoporosis. Therefore, nonlinear fracture analysis was performed under compressive conditions to simulate damage formation in the spine. The nonlinear analysis was performed by using a load increment of $150 \mathrm{~N}$ and starting from 0 to $1500 \mathrm{~N}$. During each load increment, fracture analysis was conducted on the basis of the damage model. In the damage model, two types of compressive failure were considered: plastic deformation and fracture. The assumption was that, initially, when the Drucker-Prager equivalent stress reaches the yielding stress, plastic deformation starts. Later, when the minimum principal strain reaches a critical value of $-10,000 \mu \varepsilon$, the compressive fracture occurs [21]. For each load value, Drucker-Prager stress and minimum principal strain distributions that stipulate the fracture standard were analyzed.

\section{RESULTS}

\subsection{Static Elastic Analysis during Flexion and Extension}

Static elastic analysis was performed under flexion and extension conditions, and only the Drucker-Prager stress distribution on the inferior surface of L3 vertebra was shown (Figure 4). During both load 


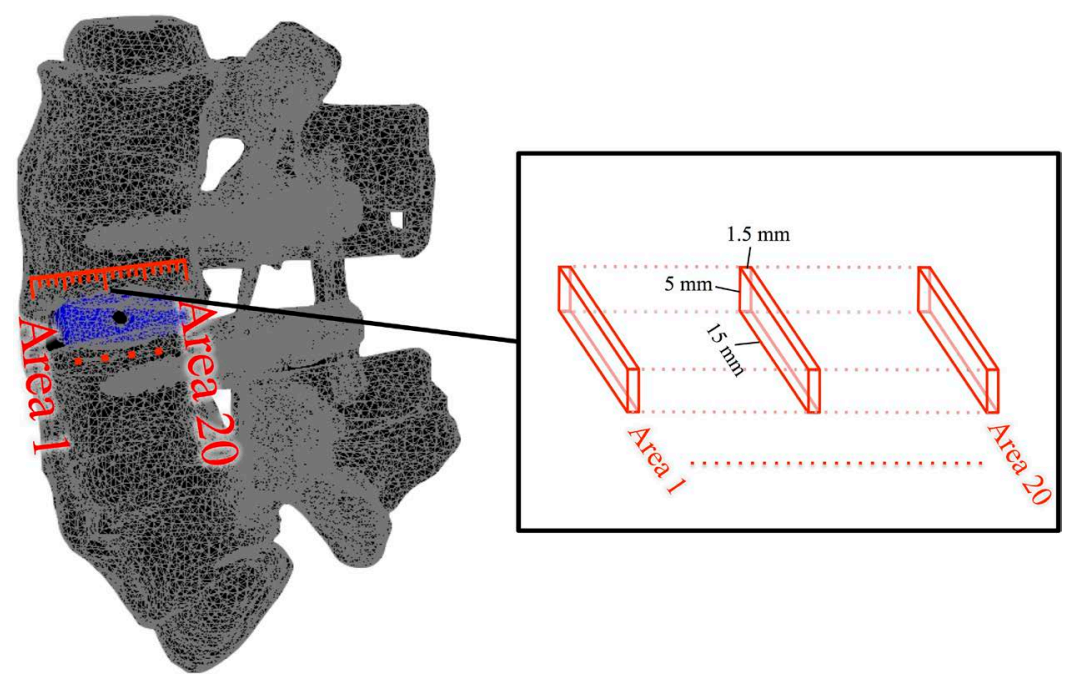

Figure 3. Each area of the bone surrounding the spacer. The bones around the spacer in L3 were divided into 20 equal areas (areas 1 to 20, each with thickness, width, and depth of $1.5 \mathrm{~mm}, 15 \mathrm{~mm}$, and $5 \mathrm{~mm}$, respectively).

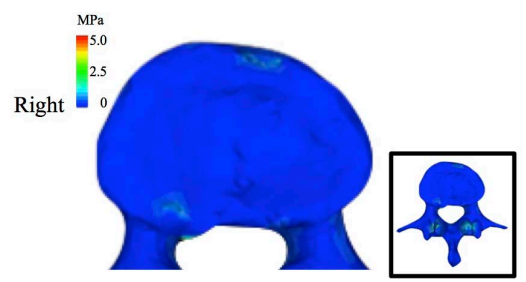

(A)

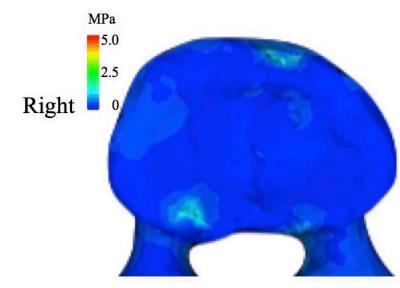

(D)

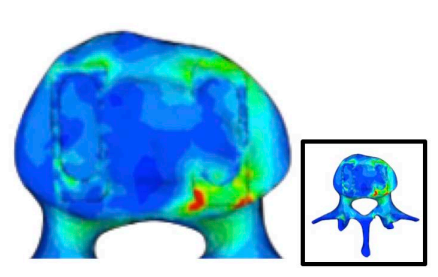

(B)

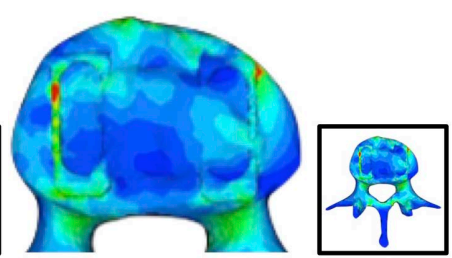

(E)

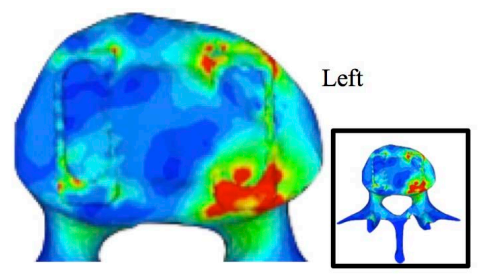

(C)

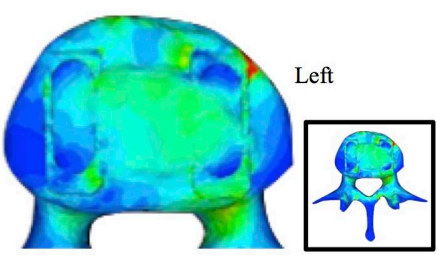

$(\mathrm{F})$

Figure 4. Each area of the bone surrounding the spacer; The Drucker-Prager stress distribution on the inferior surface of L3 vertebra. Flexion: (A) LS-INT, (B) LS-PEEK, (C) LS-Titanium; Extension: (D) LS-INT, (E) LS-PEEK, (F) LS-Titanium.

conditions, the intensity of Drucker-Prager stress in the PLIF models (LS-PEEK and LS-Titanium) was clearly higher than that of LS-INT. It was also observed that the intensity and range of Drucker-Prager stress for LS-Titanium were higher than those for LS-PEEK and tended to concentrate around the spacer, especially the spacer on the left side.

The mean values of Drucker-Prager stress, SED, and minimum principal strain in areas 1 to 20 of the bones around the L3 left spacer under flexion and extension conditions were obtained, and a significant difference was identified between the strain values of LS-PEEK and LS-Titanium (Table 2). In the flexion condition, the PLIF models (LS-PEEK and LS-Titanium) had significantly higher Drucker-Prager stress and SED and significantly lower minimum principal strain than LS-INT. Comparison between LS-PEEK and LS-Titanium showed that the latter had significantly higher Drucker-Prager stress and SED 
Table 2. Mean value of each mechanical index (Drucker-Prager stress, strain energy density, minimum principal strain) of the 20 equal areas (areas 1 to 20) of the inferior surface of L3 vertebra contacting the spacer under load conditions of flexion and extension. Models with the PEEK spacer (LS-PEEK) and the titanium spacer (LS-Titanium) were statistically compared.

\begin{tabular}{|c|c|c|c|c|}
\hline & \multirow{2}{*}{ (a) LS-INT } & \multicolumn{3}{|c|}{ PLIF model } \\
\hline & & (b) LS-PEEK & (c) LS-Titanium & $P$ value \\
\hline \multicolumn{5}{|l|}{ ( II ) Flexion } \\
\hline Drucker-Prager stress (MPa) & $0.17 \pm 0.093$ & $1.4 \pm 0.74$ & $2.7 \pm 1.5$ & $0.002^{\star *}$ \\
\hline Strain energy density $\left(\mathrm{KJ} / \mathrm{m}^{3}\right)$ & $0.00078 \pm 0.0016$ & $0.055 \pm 0.062$ & $0.25 \pm 0.25$ & $0.003^{\star *}$ \\
\hline Maximum principal stress (MPa) & $0.10 \pm 0.059$ & $0.76 \pm 0.47$ & $1.2 \pm 1.3$ & 0.12 \\
\hline $\begin{array}{l}\text { Minimum principal strain } \\
\qquad(\times 1.0 \mathrm{E}-4)\end{array}$ & $-0.026 \pm 0.035$ & $-0.28 \pm 0.30$ & $-0.65 \pm 0.43$ & $0.003^{\star *}$ \\
\hline \multicolumn{5}{|l|}{ (III) Extension } \\
\hline Drucker-Prager stress (MPa) & $0.23 \pm 0.14$ & $1.0 \pm 0.29$ & $1.5 \pm 0.37$ & $<0.001^{\star *}$ \\
\hline Strain energy density $(\mathrm{KJ} / \mathrm{m} 3)$ & $0.0018 \pm 0.0031$ & $0.035 \pm 0.047$ & $0.076 \pm 0.088$ & 0.076 \\
\hline Maximum principal stress $(\mathrm{MPa})$ & $0.047 \pm 0.10$ & $0.27 \pm 0.20$ & $0.70 \pm 0.32$ & $<0.001^{\star *}$ \\
\hline Minimum principal strain & $-0.083 \pm 0.046$ & $-0.35 \pm 0.10$ & $-0.43 \pm 0.11$ & $0.027^{\star}$ \\
\hline
\end{tabular}

${ }^{\star}$ Statistical significance $(P<0.05) .{ }^{*}$ Statistical significance $(P<0.01)$.

and significantly lower minimum principal strain than the former (Figure 5(A)). In the extension condition, the PLIF models had significantly higher Drucker-Prager strain and SED and significantly lower minimum principal strain. A comparison between LS-PEEK and LS-Titanium showed that the latter had significantly higher Drucker-Prager stress than the former, tended to have higher SED in all areas (although not a significantly higher mean value), and had significantly lower minimum principal strain (Figure 5(B)).

\subsection{Nonlinear Fracture Analysis}

As a result of the axial load increment between 0 and $1500 \mathrm{~N}$, failure occurred during the distribution state of vertical loads of $600 \mathrm{~N}$ or higher (Figure 6(A)). Compared to LS-INT, in PLIF models (LS-PEEK and LS-Titanium), failure was concentrated in the rear of the L3 vertebra around the spacer starting from an early stage of load increments. L4 vertebra had only a small number of failure elements. When examining the failure element distribution, the difference between LS-PEEK and LS-Titanium was not clear; however, the number of compressive yielding and failure elements of L3 vertebra increased with the load increment. In addition, LS-Titanium tended to constantly have a higher number of compressive yielding and failure elements than LS-PEEK (Figure 6(B) \& Figure 6(C)). The distributions of Drucker-Prager stress and minimum principal strain on the inferior surface of L3 vertebra are shown in Figure 4(D) and Figure 4(E), respectively. In PLIF models (LS-PEEK and LS-Titanium), the Drucker-Prager stress and minimum principal strain started to concentrate in the rear of L3 vertebra, around the point of contact with the spacer, from an earlier stage of load increment than in the LS-INT model. The area of concentration coincided with the points of plastic deformation and fracture.

\section{DISCUSSION}

FE analysis has been considered a sophisticated simulation method and an effective tool for elucidating biomechanics in the spine. Biomechanical evaluations based on FE analysis have shown that it is important to establish a model that can accurately reproduce the mechanical properties of each part. 
(i)

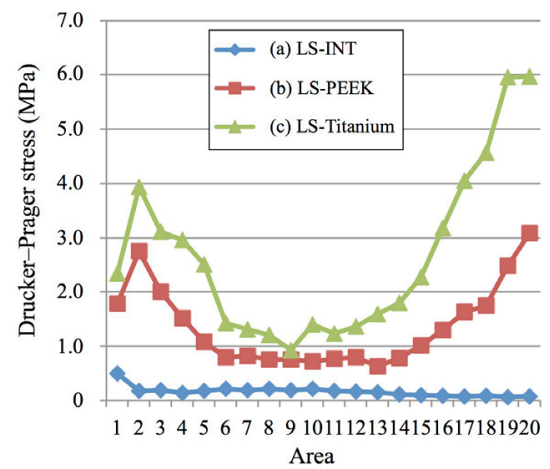

(ii )

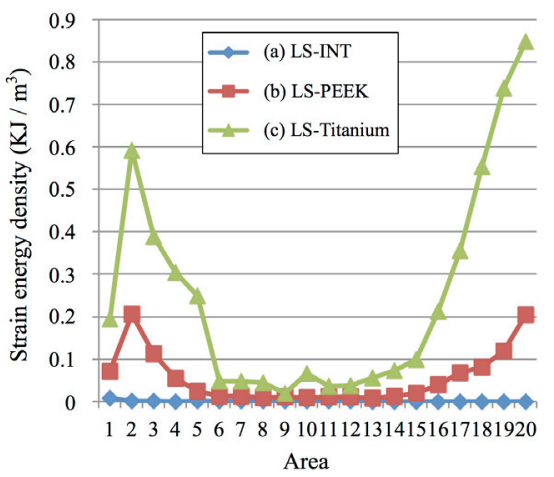

(iii)

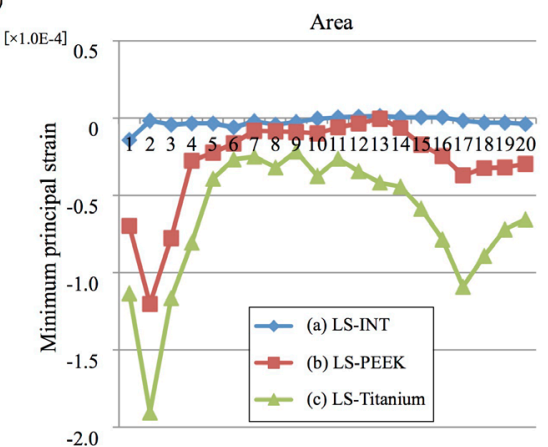

( i )

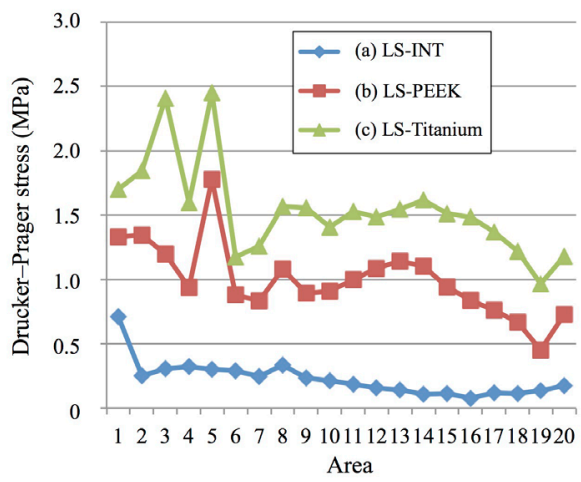

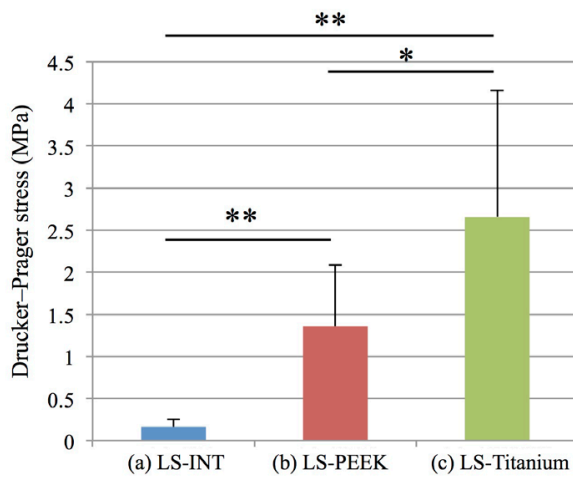

(a) LS-INT

(b) LS-PEEK

(c) LS-Titanium

(a) LS-INT

(b) LS-PEEK

(c) LS-Titanium

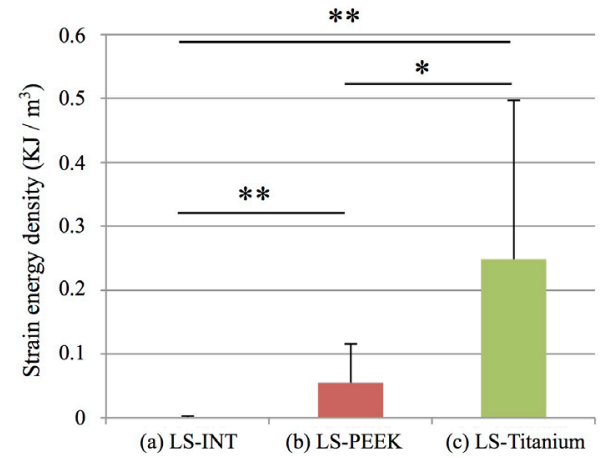

n.s.: not significant $\quad *$ p $<0.05 \quad * * p<0.01$

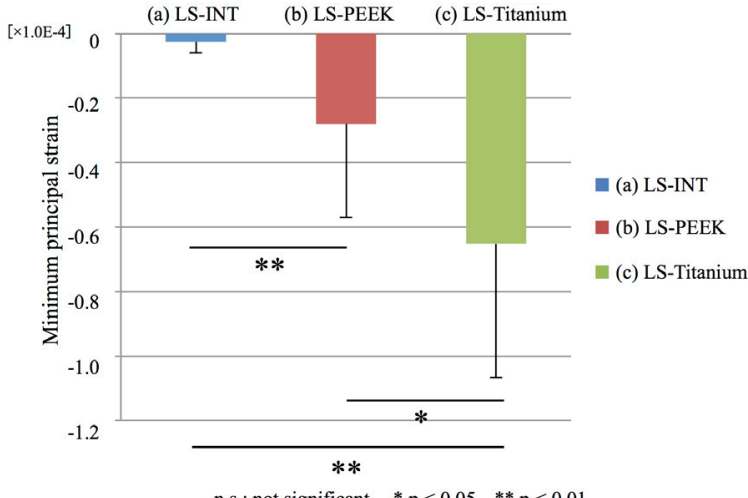

(A)

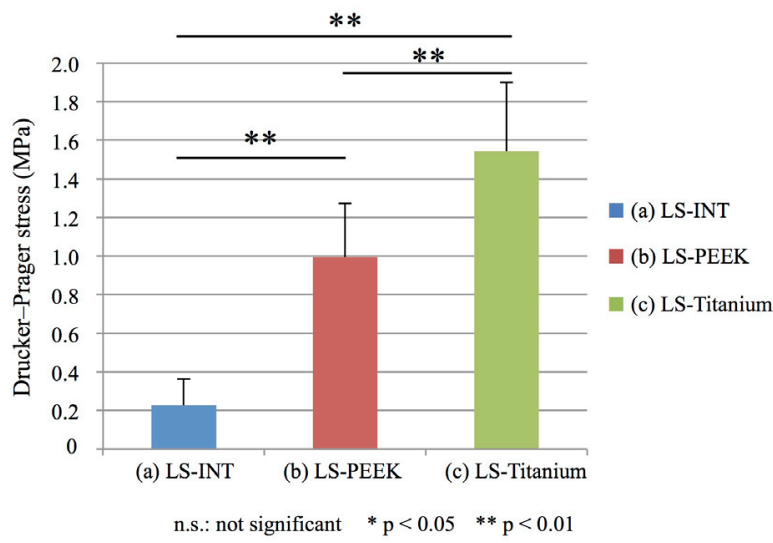



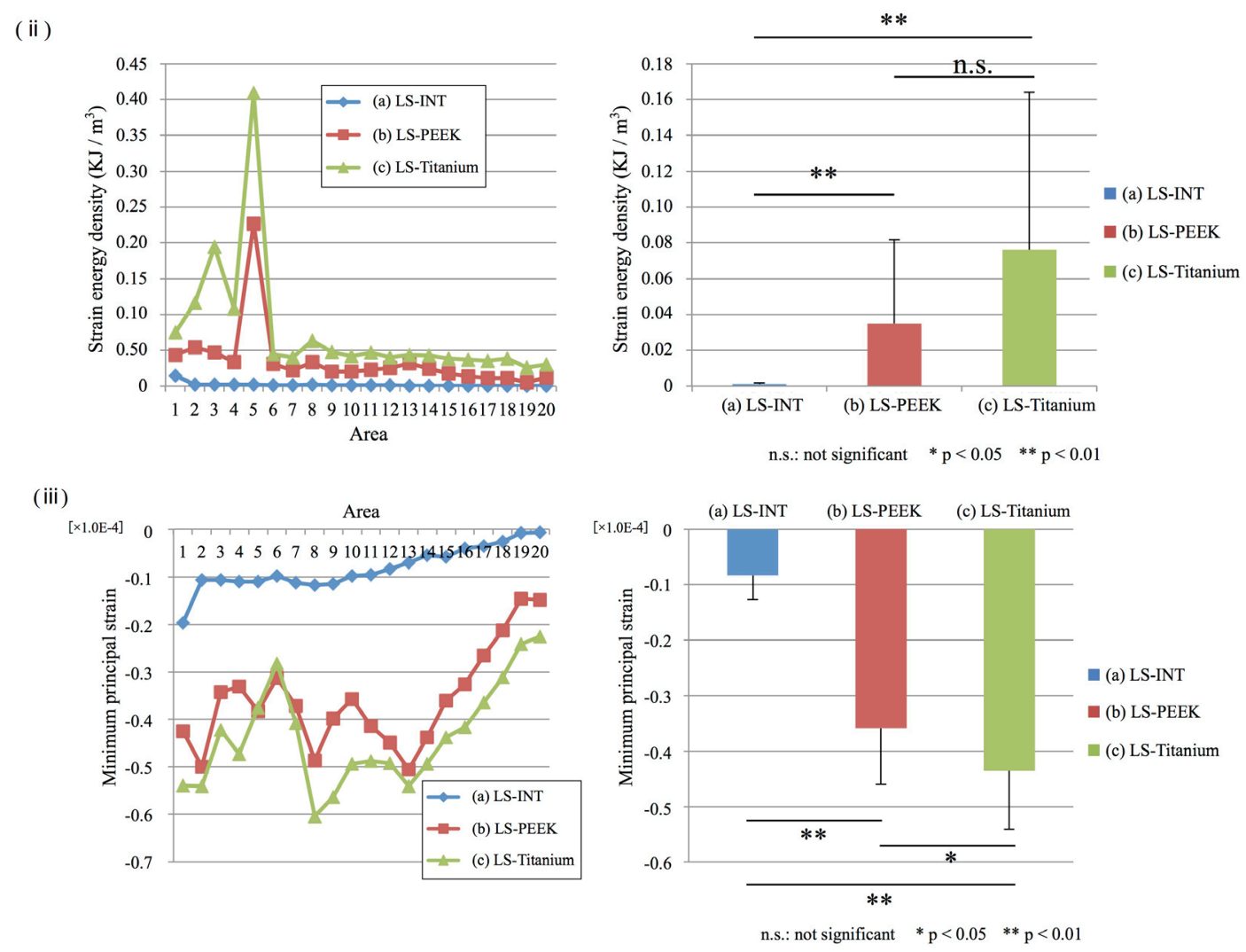

(B)

Figure 5. (A) (i) Drucker-Prager stress, (ii) strain energy density, and (iii) minimum principal strain distributions on the L3 inferior endplate of the 20 equal areasunder flexion conditions; (B) (i) Drucker-Prager stress, (ii) strain energy density, and (iii) minimum principal strain distributions on the $\mathrm{L} 3$ inferior endplate of the 20 equal areasunder extension conditions.

Establishing such a model requires patient-specific data regarding anatomic structures and material properties [22]. For this research, PLIF models were constructed by using an osteoporotic vertebra model using DICOM data of patient-specific CT scans and implants was constructed, and comparisons of the mechanical behavior of each model indicated that, for PLIF, the use of the PEEK spacer is mechanically more advantageous than the use of a titanium spacer.

Currently, both PEEK and titanium spacers are widely used for PLIF, and biomechanical studies of PLIF are still being conducted [23]. Previous reports have demonstrated the superiority of PEEK spacers through the application of FE analysis with the use of theoretical values of morphological and mechanical properties [10] [13] [14]. However, because PLIF is a type of surgery performed for elderly patients that has a clinically high risk of spacer subsidence in patients with osteoporosis [24] [25] [26] [27], it was considered necessary to perform an analysis of osteoporotic vertebrae that considered aging to demonstrate mechanical evidence and clinical applications [15]. Spacer subsidence, which is the cause of poor initial stability during interbody fusion, originates from directly below the vertebra contacting the spacer [28]. This is new information regarding the mechanical behavior of the peripheral region of the spacer.

It was clearly shown from the fracture analysis that the distribution of element failure under compression led to new mechanical knowledge that, compared to LS-INT, failure during the PLIF models (LS-PEEK and LS-Titanium) originates from the inferior surface of L3 vertebra around the spacer. This was seen as a justification to evaluate the L3 vertebrae, which are in the highest mechanical state, and especially the inferior surface of the L3 vertebra (Figure 4(A)). According to the distribution of failure 
$\Delta$ Compressive yielding elements $\Delta$ Compressive failure elements

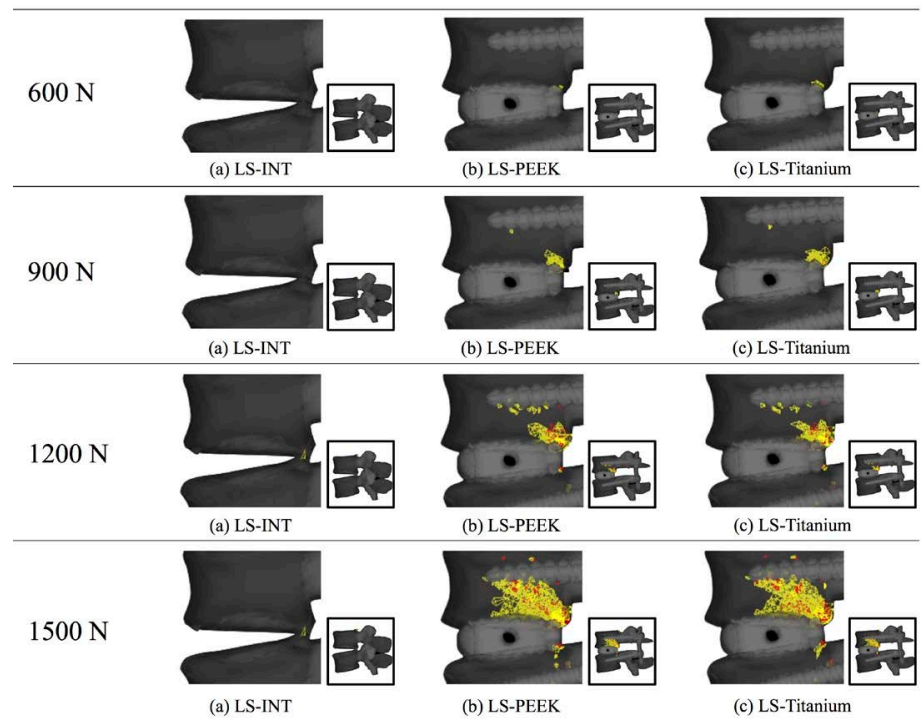

(A)

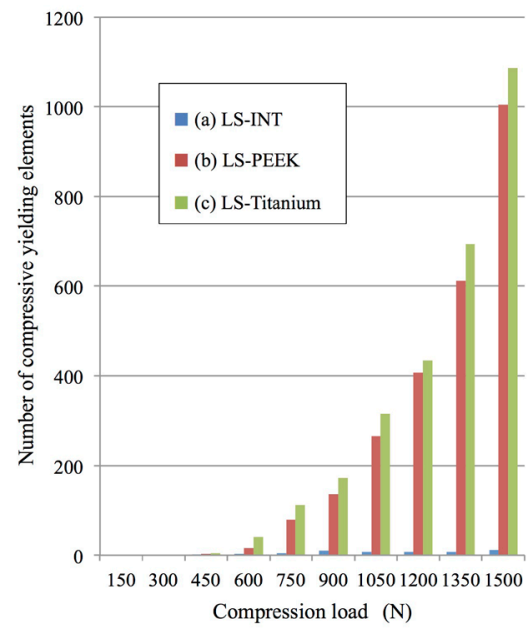

(B)

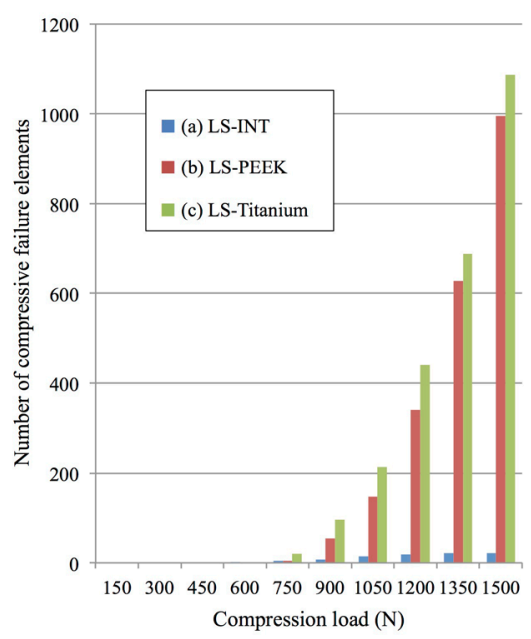

(C) 


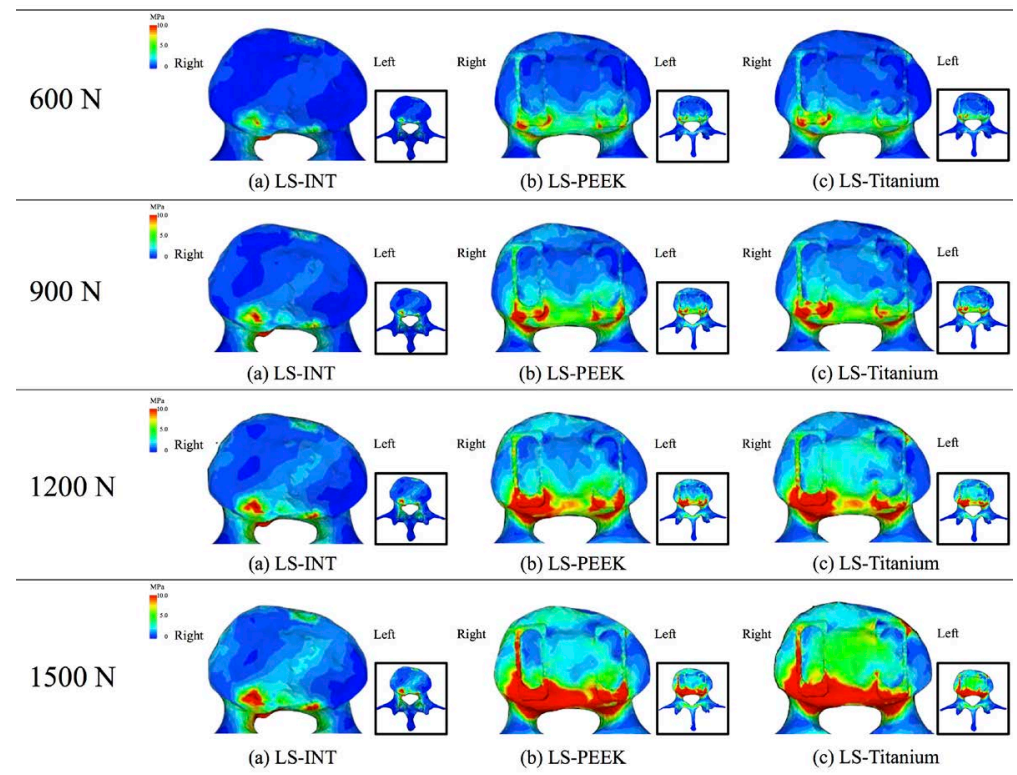

(D)

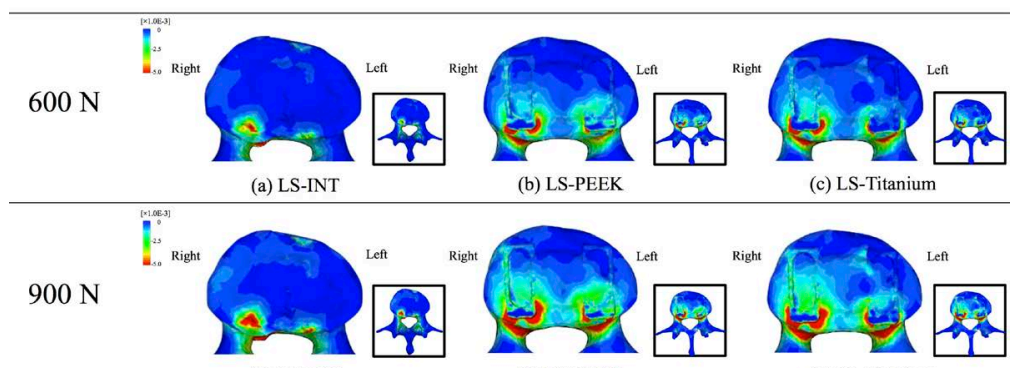

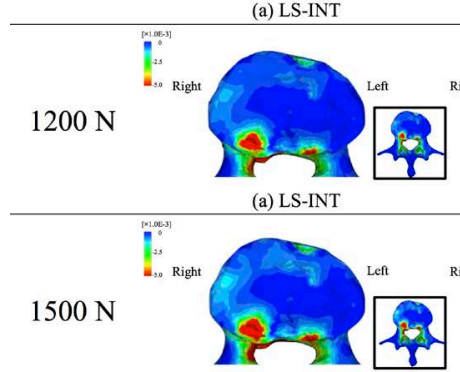

(a) LS-INT

(b) LS-PEEK (c) LS-Titanium

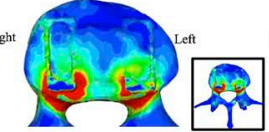
(b) LS-PEEK

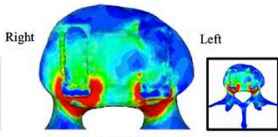
(c) LS-Titanium

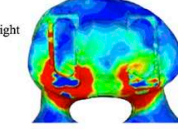

(b) LS-PEEK

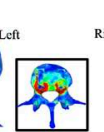

(E)

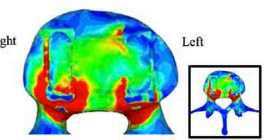

(c) LS-Titanium

Figure 6. (A) Distribution of yielding elements and failure elements under loads of 600 to $1500 \mathrm{~N}$; (B) Number of compressive yielding elementsin the L3 vertebra; (C) Number of compressive failure elements in the L3 vertebra; (D) Distribution of Drucker-Prager stress on the L3 inferior endplate under loads of 600 to $1500 \mathrm{~N}$; (E) Distribution of minimum principal strain on the L3 inferior endplate under loads of 600 to $1500 \mathrm{~N}$.

elements, the difference between LS-PEEK and LS-Titanium was not clear; however, the numbers of compressive yielding and failure elements in L3 vertebra were constantly higher for LS-Titanium than for LS-PEEK (Figure 4(B) \& Figure 4(C)). This suggested that, compared to LS-PEEK, LS-Titanium is in a mechanical environment that causes loss of bone support mechanisms due to microfractures. The distributions of Drucker-Prager stress and minimum principal strain, which stipulate the fracture criteria, showed expansion from the rear of L3 vertebra in contact with the spacer as the load was incrementally 
increased. This led to a damage mechanism involving plastic deformation and fracture elements expanding from the rear of L3 vertebra in contact with the spacer (Figure 4(D) \& Figure 4(E)).

Drucker-Prager stress exerted on the inferior surface of L3 vertebra under the conditions of flexion and extension in LS-Titanium tended to have a wider range and was higher than that of LS-PEEK (Figure 5). This Drucker-Prager stress distribution led to a hypothesis that the use of a titanium spacer, compared to a PEEK spacer, would result in stress concentration on the vertebra endplate that could more easily become an excessive load, thereby causing microfractures of the bone tissue.

Evaluation of the bone areas around the spacer under flexion and extension conditions identified a significant difference between LS-PEEK and LS-Titanium (Table 2). Evaluations of SED and of Drucker-Prager stress under flexion and extension conditions indicated that PLIF models (LS-PEEK and LS-Titanium) had higher values than did LS-INT, especially LS-Titanium (Figure 6(A-i) \& Figure 6(B-i)), thereby supporting our hypothesis. It was considered that the high mechanical stimuli in PLIF models (LS-PEEK and LS-Titanium) may negatively influence the bone remodeling process [29] [30]. Because LS-Titanium has higher mechanical stimuli, the use of a titanium spacer was thought to have a considerably negative influence on the remodeling of bones around the spacer. Similarly, during evaluation of the minimum principal strain, the PLIF models (LS-PEEK and LS-Titanium) also had smaller values than LS-INT, especially LS-Titanium (Figure 6(A-iii) \& Figure 6(B-iii)), again supporting our hypothesis.

To conclude, using a titanium spacer under conditions of compression with axial load increments from 0 to $1500 \mathrm{~N}$, nonlinear fracture analysis detected more failure elements in the vertebra. Moreover, using a titanium spacer, the influences of Drucker-Prager stress, SED, and minimum principal strain on bones around the spacer under flexion and extension conditions were stronger. However, the level of influence under stress conditions such as these is unclear. Furthermore, it was suggested that with the use of a titanium spacer, microfractures occur more easily than with a PEEK spacer, thus indicating a significantly negative influence on the bone fusion process. In other words, from a biomechanical point of view, when a titanium spacer is used for PLIF in osteoporotic vertebrae, spacer subsidence occurs more easily than with a PEEK spacer, suggesting that it is unfavorable for bone fusion. However, with the boundary conditions of this research, it is not possible to express physiological motions of the spine. Considering the complex mechanical states that must exist within a living body, further biomechanical studies of several conditions may be necessary. Previous clinical research has demonstrated that the use of PEEK spacers for lumbar interbody fusion techniques produces high fusion rates [31]. Conversely, however, some reports have stated that the use of PEEK spacers is disadvantageous for bone fusion [32]. Others have affirmed that the type of spacer makes no difference in bone fusion rates [9]. Furthermore, a report has stater that PEEK and titanium spacers were associated with similar rate of fusion, but there is an increased rate of subsidence with titanium spacers [33].Therefore, a single point of view has not yet been defined.

Reports such as this, which use FE methods built from the CT data of patients, are also used to understand the conditions of osteoporotic patients [34] and to evaluate surgery methods [18]. Therefore, their application to clinical problems is expected to grow in the future. However, analyses performed using FE have the following limitations. The load constraints of the vertebrae may be different than those of the human body during movement of the entire spine. The biological and biochemical reactions between biomaterial and bone during the fusion process have not been considered. Degenerative changes in the intervertebral disks and muscles after surgery as well as the influence of fatigue caused by repeated loads have not been considered.

\section{CONCLUSION}

Using FE analysis with a model built from clinical CT images, using patient-specific osteoporotic vertebrae data, and considering microfractures, it was possible to perform a more realistic mechanical study of vertebral column instrumentation. This research showed that PEEK spacers for PLIF in osteoporotic vertebrae compared to titanium spacers are in a mechanical environment less susceptible to subsidence caused by microfractures of the bone tissue and bone remodeling-related fusion. Therefore, the superior 
usability of PEEK spacers was suggested.

\section{REFERENCES}

1. Cloward, R.B. (1953) The Treatment of Ruptured Lumbar Intervertebral Discs by Vertebral Body Fusion. I. Indications, Operative Technique, after Care. Journal of Neurosurgery, 10, 154-168. https://doi.org/10.3171/jns.1953.10.2.0154

2. Brantigan, J.W., McAfee, P.C., Cunningham, B.W., Wang, H. and Orbegoso, C.M. (1994) Interbody Lumbar Fusion Using a Carbon Fiber Cage Implant versus allograft Bone: An Investigational Study in the Spanish Goat. Spine, 19, 1436-1444. https://doi.org/10.1097/00007632-199407000-00002

3. Brantigan, J.W. and Steffee, A.D. (1993) A Carbon Fiber Implant to Aid Interbody Lumbar Fusion: Two-Year Clinical Results in the First 26 Patients. Spine, 18, 2106-2117. https://doi.org/10.1097/00007632-199310001-00030

4. Matge, G. (2002) Cervical Cages Fusion with 5 Different Implants: 250 Cases. Acta Neurochirurgica, 144, 539-549. https://doi.org/10.1007/s00701-002-0939-0

5. Ray, C.D. (1997) Threaded Titanium Cages for Lumbar Interbody Fusions. Spine, 22, 667-679. https://doi.org/10.1097/00007632-199703150-00019

6. Eck, K.R., Bridwell, K.H., Ungacta, F.F., Lapp, M.A., Lenke, L.G. and Riew, K.D. (2000) Analysis of Titanium Mesh Cages in Adults with Minimum Two-Year Follow-Up. Spine, 25, 2407-2415. https://doi.org/10.1097/00007632-200009150-00023

7. Kuslich, S.D., Danielson, G., Dowdle, J.D., Sherman, J., Fredrickson, B., Yuan, H. and Griffith, S.L. (2000) Four-Year Follow-Up Results of Lumbar Spine Arthrodesis Using the Bagby and Kuslich Lumbar Fusion Spacer. Spine, 25, 2656-2662. https://doi.org/10.1097/00007632-200010150-00018

8. McAfee, P.C. (1999) Interbody Fusion Spacers in Reconstructive Operations on the Spine. The Journal of Bone and Joint Surgery-American Volume, 81, 859-880. https://doi.org/10.2106/00004623-199906000-00014

9. Nemoto, O., Asazuma, T., Yato, Y., Imabayashi, H., Yasuoka, H. and Fujikawa, A. (2014) Comparison of Fusion Rates Following Transforaminal Lumbar Interbody Fusion Using Polyetheretherketone Cages or Titanium Cages with Transpedicular Instrumentation. European Spine Journal, 23, 2150-2155. https://doi.org/10.1007/s00586-014-3466-9

10. Wang, Z., Fu, S., Wu, Z.X., Zhang, Y. and Lei, W. (2013) Ti2448 Pedicle Screw System Augmentation for Posterior Lumbar Interbody Fusion. Spine, 38, 2008-2015. https://doi.org/10.1097/BRS.0b013e3182a76fec

11. Couvertier, M., Germaneau, A., Saget, M., Dupré, J.C., Doumalin, P., Brémand, F., Hesser, F., Brèque, C., Roulaud, M., Monlezun, O., Vendeuvre, T. and Rigoard, P. (2017) Biomechanical Analysis of the Thoracolumbar Spine under Physiological Loadings: Experimental Motion Data Corridors for Validation of Finite Element Models. Proceedings of the Institution of Mechanical Engineers. Part H, 231, 975-981.

https://doi.org/10.1177/0954411917719740

12. Lee, C.H., Landham, P.R., Eastell, R., Adams, M.A., Dolan, P. and Yang, L. (2017) Development and Validation of a Subject-Specific Finite Element Model of the Functional Spinal Unit to Predict Vertebral Strength. Proceedings of the Institution of Mechanical Engineers. Part H, 231, 821-830. https://doi.org/10.1177/0954411917708806

13. Vadapalli, S., Sairyo, K., Goel, V.K., Robon, M., Biyani, A., Khandha, A. and Ebraheim, N.A. (2006) Biomechanical Rationale for Using Polyetheretherketone (PEEK) Spacers for Lumbar Interbody Fusion-A Finite Element Study. Spine, 31, E992-998. https://doi.org/10.1097/01.brs.0000250177.84168.ba

14. Xiao, Z., Wang, L., Gong, H. and Zhu, D. (2012) Biomechanical Evaluation of Three Surgical Scenarios of Post- 
erior Lumbar Interbody Fusion by Finite Element Analysis. BioMedical Engineering OnLine, 11, 31. https://doi.org/10.1186/1475-925X-11-31

15. Lu, Y., Rosenau, E., Paetzold, H., Klein, A., Püschel, K., Morlock, M.M. and Huber, G. (2013) Strain Changes on the Cortical Shell of Vertebral Bodies due to Spine Ageing: A Parametric Study Using a Finite Element Model Evaluated by Strain Measurements. Proceedings of the Institution of Mechanical Engineers. Part H, 227, 1265-1274. https://doi.org/10.1177/0954411913501293

16. Imai, K., Ohnishi, I., Bessho, M. and Nakamura, K. (2006) Nonlinear Finite Element Model Predicts Vertebral Bone Strength and Fracture Site. Spine, 31, 1789-1794. https://doi.org/10.1097/01.brs.0000225993.57349.df

17. Keyak, J.H., Meagher, J.M., Skinner H.B. and Mote Jr., C.D. (1990) Automated Three-Dimensional Finite Element Modelling of Bone: A New Method. Journal of Biomedical Engineering, 12, 389-397. https://doi.org/10.1016/0141-5425(90)90022-F

18. Matsuura, Y., Giambini, H., Ogawa, Y., Fang, Z., Thoreson, A.R., Yaszemski, M.J., Lu, L. and An, K.N. (2014) Specimen-Specific Nonlinear Finite Element Modeling to Predict Vertebrae Fracture Loads after Vertebroplasty. Spine, 39, E1291-1296. https://doi.org/10.1097/BRS.0000000000000540

19. Keyak, J.H., Rossi, S.A., Jones, K.A. and Skinner, H.B. (1998) Prediction of Femoral Fracture Load Using Automated Finite Element Modeling. Journal of Biomechanics, 31, 125-133. https://doi.org/10.1016/S0021-9290(97)00123-1

20. Tsuang, Y.H., Chiang, Y.F., Hung, C.Y., Wei, H.W., Huang, C.H. and Cheng, C.K. (2009) Comparison of Cage Application Modality in Posterior Lumbar Interbody Fusion with Posterior Instrumentation-A Finite Element Study. Medical Engineering \& Physics, 31, 565-570. https://doi.org/10.1016/j.medengphy.2008.11.012

21. Bessho, M., Ohnishi, I., Matsuyama, J., Matsumoto, T., Imai, K. and Nakamura, K. (2007) Prediction of Strength and Strain of the Proximal Femur by a CT-Based Finite Element Method. Journal of Biomechanics, 40, 1745-1753. https://doi.org/10.1016/j.jbiomech.2006.08.003

22. Kurtz, S.M. and Devine, J.N. (2007) PEEK Biomaterials in Trauma, Orthopedic, and Spinal Implants. Biomaterials, 28, 4845-4869. https://doi.org/10.1016/j.biomaterials.2007.07.013

23. Pelletier, M.H., Cordaro, N., Punjabi, V.M., Waites, M., Lau, A. and Walsh, W.R. (2016) PEEK versus Ti Interbody Fusion Devices: Resultant Fusion, Bone Apposition, Initial and 26-Week Biomechanics. Clinical Spine Surgery, 29, E208-E214. https://doi.org/10.1097/BSD.0b013e31826851a4

24. Oxland, T.R., Lund, T., Jost, B., Cripton, P., Lippuner, K., Jaeger, P. and Nolte, L.P. (1996) The Relative Importance of Vertebral Bone Density and Disc Degeneration in Spinal Flexibility and Interbody Implant Performance. Spine, 21, 2558-2569. https://doi.org/10.1097/00007632-199611150-00005

25. Boden, S. and Sumner, D. (1995) Biologic Factors Affecting Spinal Fusion and Bone Regeneration. Spine, 20, S102-S112. https://doi.org/10.1097/00007632-199512151-00007

26. Sethi, A., Lee, S. and Vaidya, R. (2009) Transforaminal Lumbar Interbody Fusion using Unilateral Pedicle Screws and a Translaminar Screw. European Spine Journal, 18, 430-434.

https://doi.org/10.1007/s00586-008-0825-4

27. Oh, K.W., Lee, J.H., Lee, D.Y. and Shim, H.J. (2017) The Correlation between Cage Subsidence, Bone Mineral Density, and Clinical Results in Posterior Lumbar Interbody Fusion. Clinical Spine Surgery, 30, E683-E689. https://doi.org/10.1097/BSD.0000000000000315

28. Lee, J.H., Jeon, D.W., Lee, S.J., Chang, B.S. and Lee, C.K. (2010) Fusion Rates and Subsidence of Morselized Local Bone Grafted in Titanium Cages in Posterior Lumbar Interbody Fusion using Quantitative Three-Dimensional Computed Tomography Scans. Spine, 35, 1460-1465.

https://doi.org/10.1097/BRS.0b013e3181c4baf5 
29. Herrera, A., Panisello, J.J., Ibarz, E., Cegoñino, J., Puértolas, J.A. and Gracia, L. (2009) Comparison between DEXA and Finite Element Studies in the Long-Term Bone Remodeling of an Anatomical Femoral Stem. Journal of Biomechanical Engineering, 131, Article ID: 041013. https://doi.org/10.1115/1.3072888

30. Huiskes, R., Weinans, H., Grootenboer, H.J., Dalstra, M., Fudala, B. and Slooff, T.J. (1987) Adaptive Bone-Remodeling Theory Applied to Prosthetic-Design Analysis. Journal of Biomechanics, 20, 1135-1150. https://doi.org/10.1016/0021-9290(87)90030-3

31. Lee, J.H., Lee, J.H., Park, J.W. and Lee, H.S. (2011) Fusion Rates of a Morselized Local Bone Graft in Polyetheretherketone Spacers in Posterior Lumbar Interbody Fusion by Quantitative Analysis using Consecutive Three-Dimensional Computed Tomography Scans. The Spine Journal, 11, 647-653. https://doi.org/10.1016/j.spinee.2011.04.029

32. Schimmel, J.J., Poeschmann, M.S., Horsting, P.P., Schönfeld, D.H., van Limbeek, J. and Pavlov, P.W. (2016) Comparison between DEXA and Finite Element Studies in the Long-Term Bone Remodeling of an Anatomical Femoral Stem. Clinical Spine Surgery, 29, E252-E258. https://doi.org/10.1097/BSD.0b013e31826eaf74

33. Seaman, S., Kerezoudis, P., Bydon, M., Torner, J.C. and Hitchon, P.W. (2017) Titanium vs. Polyetheretherketone (PEEK) Interbody Fusion: Meta-Analysis and Review of the Literature. Journal of Clinical Neuroscience, 44, 23-29. https://doi.org/10.1016/j.jocn.2017.06.062

34. Tawara, D., Sakamoto, J., Murakami, H., Kawahara, N., Oda, J. and Tomita, K. (2010) Mechanical Therapeutic Effects in Osteoporotic L1-Vertebrae Evaluated by Nonlinear Patient-Specific Finite Element Analysis. Journal of Biomechanical Science and Engineering, 5, 499-514. https://doi.org/10.1299/jbse.5.499 